Revista Brasileira de Agricultura Irrigada v.5, nº. 3, p 165-172., 2011

ISSN 1982-7679 (On-line)

Fortaleza, CE, INOVAGRI - http://www.inovagri.org.br

Protocolo 05511 - 18/04/2011 Aprovado em 06/09/2011

\title{
AVALIAÇÃO AGRONÔMICA DE CULTIVARES DE SOJA SOB IRRIGAÇÃO NO SEMIÁRIDO MINEIRO
}

Leonardo Angelo de Aquino ${ }^{1}$, Tatiane Carla Silva ${ }^{2}$, Rosiane Filomena Batista Almeida de Aquino $^{3}$, Carlos Henrique Batista ${ }^{2}$, Heider Rodrigo Ferreira Silva ${ }^{2}$

\footnotetext{
${ }^{1}$ Engenheiro Agrônomo, D. Sc. Fitotecnia, Professor Adjunto, Universidade Federal de Viçosa (UFV) Campus Rio Paranaíba. MG230, km 08, CEP 38.810-000. Fone: (34) 3855 9353. E-mail: leonardo.aquino@ufv.br

2 Acadêmico de Agronomia, Instituto Federal de Educação, Ciência e Tecnologia do Norte de Minas Campus Januária. Fazenda São Geraldo, s/n, Bom Jardim, CEP 39480-000, Januária, MG. E-mail: tatianeagronomia@gmail.com; carlosbatista.agro@yahoo.com.br; heiderrfs@yahoo.com.br.

3 Acadêmico de Agronomia, UFV - Campus Rio Paranaíba. MG230, km 08, CEP 38.810-000.

Fone: (34) 3855 9353. E-mail: rosiane.aquino@yahoo.com.br
}

RESUMO: O alcance de produtividade satisfatória da cultura da soja parte da escolha adequada das cultivares de acordo com as condições edafoclimáticas da região de cultivo. Objetivou-se, nesse trabalho, avaliar aspectos agronômicos de cultivares de soja sob condições de irrigação no semiárido mineiro. O experimento foi conduzido no Instituto Federal do Norte de Minas Gerais, Campus de Januária. Os tratamentos consistiram de oito cultivares de soja: M-SOY 9350, M-SOY 8787 RR, M-SOY 8352 RR, CD 219 RR, P98Y51, P98Y70, P99R01, P99R91. Adotou-se o delineamento em blocos casualizados com quatro repetições. Cada parcela foi composta por quatro linhas de $10 \mathrm{~m}$. Como área útil adotou-se as duas linhas centrais, menos $50 \mathrm{~cm}$ das extremidades. A instalação da cultura e os tratos culturais foram os comumente preconizados para o cultivo da soja. O experimento foi irrigado por aspersão convencional. Avaliaram-se as seguintes características: altura de inserção de primeira vagem e de plantas, número de vagens por planta, ciclo, massa de 100 sementes e produtividade de grãos. A cultivar M-SOY 8352 RR não apresentou altura de plantas adequada para a colheita mecanizada. Todas as cultivares avaliadas apresentaram altura de inserção de primeira vagem desfavorável à colheita mecanizada. A cultivar mais tardia foi a P99R01. As cultivares mais produtivas foram P98Y70 e P99R01.

Palavras chave: Glycine max (L.) Merrill; aspersão convencional; ciclo cultural.

\section{AGRONOMIC EVALUATION OF SOYBEAN CULTIVARS UNDER IRRIGATION IN SEMIARID REGION OF MINAS GERAIS STATE}

ABSTRACT: To reach soybean maximum yields, we should start from an appropriate cultivars choice according to the edaphoclimatic conditions of the growing region. This study aimed to evaluate the agronomic aspects of soybean cultivars under irrigated conditions in semiarid region of Minas Gerais. The experiment was carried out at 


\section{AVALIAÇÃO AGRONÔMICA DE CULTIVARES DE SOJA SOB IRRIGAÇÃO NO SEMIÁRIDO MINEIRO}

Instituto Federal do Norte de Minas Gerais (Federal Institute of Northern of Minas Gerais), Campus Januária. The treatments consisted of eight soybean cultivars, M-SOY 9350, M-SOY 8787 RR, M-SOY 8352 RR, CD 219 RR, P98Y51, P98Y70, P99R01, P99R91. We adopted the randomized block design with four replications. Each plot consisted of four rows of $10 \mathrm{~m}$. The center two line, less $50 \mathrm{~cm}$ from the ends, was the usable area. The used culture installation and cultural practices were the standard for soybean cultivation. The conventional sprinkler was used in the experiment. The height of first pod insertion and plant, number of pods per plant, cycle, weight of hundred seeds and grains yield characteristics were evaluated. The M-SOY 8352 RR cultivar did not reach the proper height for mechanical harvesting. All evaluated cultivars showed unfavorable height of first pod insertion to the mechanized harvesting. The later cultivar was P99R01. The more productive cultivars were P98Y70 and P99R01.

Keywords: Glycine $\max$ (L.) Merrill, conventional sprinkler, cultural cycle.

\section{INTRODUÇÃO \\ A soja (Glycine max (L.)} Merrill) é uma das mais importantes oleaginosas cultivadas no mundo. Seu elevado teor de proteína $(40 \%)$ e de óleo $(20 \%)$ associado ao alto rendimento de grãos, a torna uma alternativa importante para a alimentação animal e humana. O oléo da soja pode ser utilizado na alimentação humana e atualmente como matéria prima para produção de biocombustíveis (Sediyama et al., 2009). Estima-se para a safra 2010/11 a maior produção histórica brasileira com área cultivada de aproximadamente 24 milhões de hectares e produtividade média de $3079 \mathrm{~kg} \mathrm{ha}^{-1}$ (Conab, 2010).

Guimarães et al. (2008) afirmam que a produtividade de uma cultura é definida pela interação entre o genótipo da planta, o ambiente de produção e o manejo. A escolha de cultivares melhores adaptadas a cada região está entre as práticas de manejo mais importantes para a soja.

O fotoperíodo e a temperatura do ar do local de cultivo são variáveis meteorológicas importantes porque provocam mudanças qualitativas ao longo do ciclo da soja (Rodrigues et al., 2001). Fotoperíodos curtos ou altas temperaturas favorecem a redução do tempo necessário para o florescimento, resultando em plantas de porte baixo e poucos nós, fatores que afetam negativamente a produtividade (Câmara et al., 1997).

Devido a influência do comprimento do dia no desenvolvimento da cultura, é necessário conhecimento sobre cultivares mais adaptadas a cada região, se não, o empreendimento pode estar fadado ao insucesso (Braccini et al., 2004). O alcance de produtividades satisfatórias parte primeiramente da identificação das cultivares mais promissoras em determinada região de cultivo.

A época de semeadura ideal para soja na maior parte do território brasileiro é do mês de outubro ao de dezembro. Semeadura antes ou após a época indicada pode causar redução da produtividade devido à redução do ciclo ou deficiência hídrica em fases críticas, como no florescimento (Fidelis et al., 2007; Braccini et al., 2004). Assim, cultivares indicadas para essa época de semeadura devem ser selecionadas para cada região de cultivo.

Na região Norte de Minas Gerais existem grandes perímetros irrigados, como o de Jaíba, o de Gorotuba e o de Pirapora. Ainda existem áreas de cultivo de oleaginosas, como por exemplo, nos municípios de Chapada Gaúcha e de São Romão, com possibilidade de produção irrigada. Carece, no entanto, de adequação de tecnologias, como a 


\section{AVALIAÇÃO AGRONÔMICA DE CULTIVARES DE SOJA SOB IRRIGAÇÃO NO SEMIÁRIDO MINEIRO}

escolha de materiais genéticos adaptados às condições edafoclimáticas da referida região.

Baseado neste contexto objetivou-se, com este trabalho, avaliar o desempenho agronômico de cultivares soja sob condições irrigadas no semiárido mineiro.

\section{MATERIAL E MÉTODOS}

O trabalho foi conduzido no período de novembro de 2009 a abril de 2010, na Unidade de Pesquisa do Instituto Federal do Norte de Minas Gerais (IFNMG), Campus Januária. A Unidade fica localizada na latitude $15^{\circ}$ $28^{\prime} 55^{\prime}$ ' S e longitude $44^{\circ} 22^{\prime} 41^{\prime}$ ' W, possui altitude $474 \mathrm{~m}$ e clima Aw (tropical úmido com inverno seco $\mathrm{e}$ verão chuvoso) de acordo com a classificação de Köppen. A precipitação, a umidade relativa e a temperatura média anual, são $850 \mathrm{~mm}$, $60 \%$ e $27{ }^{\circ} \mathrm{C}$, respectivamente (Jacomine et al., 1979).

No preparo do solo procedeu-se o revolvimento com grade aradora, seguido por nivelamento e destorroamento com uma passada de grade niveladora.

O solo da área experimental, de textura arenosa, foi classificado como Neossolo Quartzarênico, cujos atributos físico-químicos são apresentados na Tabela 1.

A calagem e a adubação foram baseadas em recomendações para a cultura da soja e nos resultados da análise química do solo (Ribeiro et al., 1999). Aplicou-se no sulco de semeadura $200 \mathrm{~kg} \mathrm{ha}^{-1}$ do formulado 430-10. Este formulado continha $0,7 \%$ de B. Em cobertura, aos 25 e 40 dias após a emergência (DAE), aplicou-se $86 \mathrm{~kg}$ $\mathrm{ha}^{-1}$ de $\mathrm{KCl}$ em cada aplicação.

Tabela 1. Características químicas e físicas do solo da área experimental na camada de $0-20 \mathrm{~cm}$.

\begin{tabular}{|c|c|c|c|c|c|c|c|c|}
\hline $\begin{array}{c}\mathrm{pH} \\
\left(\mathrm{H}_{2} \mathrm{O}, 1: 2,5\right)\end{array}$ & $\begin{array}{c}\text { M.O } \\
\text { dag kg }{ }^{-1}\end{array}$ & \multicolumn{2}{|c|}{-- $\mathrm{mg} \mathrm{dm}^{-3}--$} & $\mathrm{Ca}^{2+}$ & ---------- & $\mathrm{l}_{\mathrm{c}} \mathrm{dm}^{-}$ & $\mathrm{H}+\mathrm{Al}$ & $\mathrm{T}$ \\
\hline 5,8 & 0,6 & 26,9 & 27 & 2,1 & 0,5 & 0,0 & 1,07 & 3,8 \\
\hline \multirow[t]{2}{*}{ B } & $\mathrm{Zn}$ & $\mathrm{Mn}$ & $\mathrm{Cu}$ & $\mathrm{Fe}$ & P-rem & Areia & Silte & Argila \\
\hline & ------ m & $\mathrm{dm}^{-3}--$ & ----- & & $\mathrm{mg} \mathrm{L}^{-1}$ & --- & $\mathrm{g} \mathrm{kg}^{-1}-$ & \\
\hline 0,42 & 3,5 & 31,6 & 1,3 & 18,1 & 44,4 & 75 & 12 & 13 \\
\hline
\end{tabular}

Extratores: M.O - Matéria Orgânica: método da oxidoredução; P, K, Zn, Mn, Cu e Fe: Mehlich - 1; Ca, $\mathrm{Mg}$ e $\mathrm{Al}: \mathrm{KCl} 1 \mathrm{~mol} \mathrm{~L}^{-1} ; \mathrm{H}+\mathrm{Al}: \mathrm{Ca}(\mathrm{OAc})_{2}$ 0,5 mol L ${ }^{-1}, \mathrm{pH} \mathrm{7}$; $\mathrm{B}$ : Água quente; $\mathrm{P}$ rem = Fósforo remanescente.

\begin{abstract}
Realizou-se a inoculação das sementes com Bradyrhizobium japonicum na dose de 1,2 milhão de células por semente. Utilizou-se para inoculante turfoso contendo as estirpes SEMIA 587, 5019 e 5080 de Bradyrhizobium japonicum. A semeadura foi realizada no dia $11 / 11 / 2009$, com espaçamento entre fileiras de $0,6 \mathrm{~m}$ e com sementes
\end{abstract}

suficientes para alcance da população de 250 mil plantas ha ${ }^{-1}$.

Os tratamentos consistiram de oito cultivares de soja, indicadas para cultivo no Norte de Minas Gerais e em regiões com temperaturas médias anuais acima de $25^{\circ} \mathrm{C}$. As cultivares utilizadas foram: M-SOY 9350, M-SOY 8787 RR, M-SOY 8752 RR, CD 219 RR, P98Y51, P98Y70, P99R01, P99R91. Adotou-se o delineamento em blocos 


\section{AVALIAÇÃO AGRONÔMICA DE CULTIVARES DE SOJA SOB IRRIGAÇÃO NO SEMIÁRIDO MINEIRO}

casualizados com quatro repetições. Cada parcela foi composta por quatro linhas de $10 \mathrm{~m}$. A área útil foi compreendida pelas duas fileiras centrais, menos $50 \mathrm{~cm}$ das extremidades.

O manejo de plantas daninhas consistiu da aplicação de herbicidas recomendados para a cultura aos 24 DAE. Utilizou-se $120 \mathrm{~g} \mathrm{ha}^{-1} \mathrm{de}$ Fomesafen $\left(\right.$ Flex $\left.^{\circledR}\right)$ e $100 \mathrm{~g} \mathrm{ha}^{-1}$ de fluazifop-p-butil (Fusilade $250 \mathrm{EW}^{\circledR}$ ). Juntamente com os herbicidas aplicouse $30 \mathrm{~g} \mathrm{ha}^{-1}$ de Mo via molibdato de amônio.

Realizou-se uma aplicação de Metamidafos no início de enchimento de grãos e outra de Endossulfan na maturação fisiológica para controle de percevejos (Picanço et al., 2009). No estádio de início de enchimento de grãos aplicou-se Tetraconazole $\left(\right.$ Domark $^{\circledR}$ ) para controle de doenças de final de ciclo (Dhingra et al., 2009).

A cultura foi irrigada com uso do sistema de aspersão convencional. Realizou-se avaliação da precipitação e uniformidade de aplicação do sistema (Bernardo et al., 2006). A lâmina média aplicada pelo sistema foi de $5 \mathrm{~mm} \mathrm{~h}^{-1} \mathrm{e}$ o coeficiente de uniformidade de Cristhiansen foi de $87,1 \%$. Diariamente foram registrados os dados meteorológicos em uma estação automática localizada a $300 \mathrm{~m}$ da área experimental.

Diariamente foi reposta a lâmina correspondente a evapotranspiração da cultura. Essa foi calculada pelo produto entre a evapotranspiração de referência e coeficientes de cultivo para a soja (Bernardo et al., 2006). A evapotranspiração de referência foi calculada pelo método de PenmanMonteith (Allen et al., 2006) com o uso dos dados meteorológicos coletados diariamente.

Avaliaram-se as seguintes características: Altura média das plantas (AP)- determinada a partir da superfície do solo até a extremidade do caule principal; Altura média de inserção da primeira vagem (AIPV) determinada a partir da superfície do solo até a inserção da primeira vagem; Número de vagens por planta (NVP) determinados em dez plantas aleatórias na área útil da parcela, por ocasião da plena maturação da cultura; Massa de cem grãos (MCS) - obtida por meio da contagem e pesagem de cem grãos selecionados da área útil da parcela; Produtividade de grãos (PROD) obtida após pesagem dos grãos das plantas da área útil de cada parcela $(\mathrm{kg}$ parcela $\left.^{-1}\right)$ e transformado para rendimento $\left(\mathrm{kg} \mathrm{ha}^{-1}\right)$, após ajuste da umidade para 13\%; Ciclo cultural (CC)foi computado o número de dias entre a emergência e a colheita.

A colheita foi realizada manualmente à medida que se atingiu o ponto de colheita de cada cultivar (umidade em torno de 15\%).

Os dados obtidos foram submetidos à análise de variância, com auxílio do software estatístico SAEG Versão 9.1 (Saeg, 2007). As médias dos tratamentos foram comparadas pelo critério de Scott-Knott a 5\% de probabilidade.

\section{RESULTADOS E DISCUSSÃO}

Houve diferença entre as cultivares avaliadas para altura e número de vagens por planta, massa de 100 sementes, ciclo cultural e produtividade. Não houve efeito significativo na altura de inserção da primeira vagem (Tabela 2). A precipitação total durante a condução do experimento foi de $767 \mathrm{~mm}$. O consumo hídrico total da cultura foi de $429 \mathrm{~mm}$ nos cultivares CD 219 RR, M-SOY 8787 RR, P98R31 e M-SOY8352 RR (mais precoces) e de $523 \mathrm{~mm}$ nos cultivares P98Y70, P99R01, M-SOY 9350 e P98R91 (mais tardios). A 


\section{AVALIAÇÃO AGRONÔMICA DE CULTIVARES DE SOJA SOB IRRIGAÇÃO NO SEMIÁRIDO MINEIRO}

diferença no consumo hídrico total deveu-se à diferença de ciclo dos cultivares. Apesar da precipitação pluvial superar o consumo hídrico total, foi necessário suplementar $187 \mathrm{~mm}$ nos cultivares mais precoces e $243 \mathrm{~mm}$ naqueles mais tardios. A suplementação de água via irrigação foi necessária devido à baixa capacidade de armazenamento de água do solo e à precipitação com distribuição irregular.

A média geral de altura de plantas ficou ao redor de $50 \mathrm{~cm}$. Tal valor é considerado como ideal para colheita mecanizada, desde que a declividade da área de cultivo não seja acentuada (Sediyama et al., 2009). Diversos fatores como a época de semeadura, o espaçamento de plantas entre e dentro das fileiras, o suprimento de água, a temperatura, a fertilidade do solo também podem provocar variações na altura de plantas (Guimarães et al., 2008).

No experimento adotou-se o espaçamento de $60 \mathrm{~cm}$ (Tabela 2). Para obtenção de plantas com maior altura uma opção seria o semeio com espaçamentos menores. A adoção de espaçamentos menores e de populações de plantas maiores contribui para maior altura de plantas (Endres, 1996).

Todos os cultivares apresentaram altura de inserção da primeira vagem abaixo do recomendado para colheita mecanizada (Almeida, 2008; Sediyama et al., 2009). A pequena altura de inserção de primeira vagem pode estar em parte, relacionada ao espaçamento utilizado. Maiores espaçamentos contribuem para menor altura de inserção das primeiras vagens na planta. Em parte, a reduzida altura de inserção, pode ser explicada pelas altas temperaturas durante a condução do experimento, especialmente durante $\mathrm{o}$ crescimento vegetativo da cultura. Para minimizar as perdas de colheita por pequena altura de inserção da primeira vagem é ideal que as primeiras vagens estejam a pelos menos $10 \mathrm{~cm}$ do solo (Yokomizo, 1999; Carvalho et al., 2010).

Tabela 2. Altura de inserção de primeira vagem (AIPV) e altura de plantas (AP), número de vagens por planta (NVP), massa de 100 sementes (MCS), ciclo cultural (CC) e produtividade (PROD) de cultivares de soja sob irrigação no Norte de Minas Gerais. Januária, IFNMG - Campus Januária, 2010.

\begin{tabular}{lcccccc}
\hline \multicolumn{1}{c}{ Cultivares } & $\begin{array}{c}\text { AIPV } \\
(\mathrm{cm})\end{array}$ & $\begin{array}{c}\text { AP } \\
(\mathrm{cm})\end{array}$ & $\begin{array}{c}\text { NVP } \\
(\text { unid })\end{array}$ & $\begin{array}{c}\text { MCS } \\
(\mathrm{g})\end{array}$ & $\begin{array}{c}\text { CC } \\
(\text { dias })\end{array}$ & $\begin{array}{c}\text { PROD } \\
\left(\mathrm{kg} \mathrm{ha}^{-1}\right)\end{array}$ \\
\hline P98Y70 & $7,4 \mathrm{a}$ & $53,8 \mathrm{a}$ & $101 \mathrm{~b}$ & $17,0 \mathrm{~b}$ & $127 \mathrm{~b}$ & $3.997 \mathrm{a}$ \\
P99R01 & $7,6 \mathrm{a}$ & $48,5 \mathrm{~b}$ & $128 \mathrm{a}$ & $12,3 \mathrm{e}$ & $140 \mathrm{a}$ & $3.897 \mathrm{a}$ \\
M-SOY 9350 & $7,7 \mathrm{a}$ & $50,7 \mathrm{~b}$ & $112 \mathrm{a}$ & $17,8 \mathrm{a}$ & $125 \mathrm{~b}$ & $2.558 \mathrm{~b}$ \\
P98R91 & $9,5 \mathrm{a}$ & $48,9 \mathrm{~b}$ & $113 \mathrm{a}$ & $14,5 \mathrm{~d}$ & $128 \mathrm{~b}$ & $2.353 \mathrm{~b}$ \\
M-SOY 8352 RR & $7,5 \mathrm{a}$ & $41,1 \mathrm{c}$ & $89 \mathrm{~b}$ & $16,2 \mathrm{c}$ & $120 \mathrm{c}$ & $1.892 \mathrm{c}$ \\
P98R31 & $7,7 \mathrm{a}$ & $49,7 \mathrm{~b}$ & $83 \mathrm{~b}$ & $16,1 \mathrm{c}$ & $120 \mathrm{c}$ & $1.847 \mathrm{c}$ \\
M-SOY 8787 RR & $7,8 \mathrm{a}$ & $55,9 \mathrm{a}$ & $75 \mathrm{~b}$ & $11,9 \mathrm{f}$ & $114 \mathrm{~d}$ & $1.800 \mathrm{c}$ \\
CD 219 RR & $7,3 \mathrm{a}$ & $49,9 \mathrm{~b}$ & $95 \mathrm{~b}$ & $14,5 \mathrm{~d}$ & $114 \mathrm{~d}$ & $1.742 \mathrm{c}$ \\
\hline F & $1,40^{\mathrm{ns}}$ & $6,58^{*}$ & $2,49^{*}$ & $305,65^{* * *}$ & $30,09^{* * *}$ & $35,84^{* * *}$ \\
CV (\%) & 11,7 & 6,8 & 22,5 & 1,6 & 2,5 & 13,9 \\
Média & 7,8 & 49,8 & 100 & 15,0 & 123 & 2.511 \\
\hline
\end{tabular}

${ }^{\pi 1}$ Médias seguidas por mesma letra na coluna não diferem entre si pelo critério de Scott -Knott ao nível de $5 \%$ de probabilidade.

$\mathrm{O}$ número de vagens por planta variou de 75 a 128 (Tabela 2). O maior número de vagens por planta resultou em maior produtividade das cultivares, exceto na cultivar P98Y70. O número de vagens por planta é um dos componentes de produção com maior correlação positiva com a produtividade de grão em 


\section{AVALIAÇÃO AGRONÔMICA DE CULTIVARES DE SOJA SOB IRRIGAÇÃO} NO SEMIÁRIDO MINEIRO

leguminosas (Navarro Júnior \& Costa 2002). O número de vagens por planta nas cultivares P98Y70, P99R01, MSOY 9350 e P98R91 foi semelhante aos obtidos em estudo realizado em São Desidério - BA (Cruz et al., 2010). No estudo os autores concluíram que a melhor época de semeadura foi no mês de novembro.

A massa de 100 sementes foi a característica que apresentou a maior variação entre os cultivares (Tabela 2). A cultivar M-SOY 9350 apresentou as sementes mais pesadas $(17,8 \mathrm{~g})$. No entanto, a cultivar P99R01 apesar de estar no grupo das menores médias para a característica massa de 100 sementes foi uma das cultivares mais produtivas. Não só a massa de 100 sementes, mas a também o número de vagens por planta e de grãos por vagem determinam a produtividade final da lavoura (Cruz et al., 2010).

As cultivares P98Y70 e P99R01 superaram a produtividades das demais cultivares avaliadas. Essas cultivares apresentaram produtividade média acima da média nacional na safra 2009/10 (Conab, 2010) e semelhantes as obtidas em São Desidério - BA com a semeadura realizada no mês de novembro (Cruz et al., 2010).

A cultivar P99R01 tem ciclo mais tardio que P98Y70 (Tabela 2). Cultivares mais tardias, de modo geral, apresentam menor perda de produtividade quando semeadas após a época adequada em uma determinada região, ou, em regiões de baixa latitude e sujeitas a temperaturas elevadas (Câmara et al., 1997; Sediyama et al., 2009). Assim, ambas cultivares podem ser indicadas para cultivo na regiao Norte de Minas Gerais. Sugere-se o uso de menor espaçamento para estimular maior altura de plantas e de inserção de primeira vagem.

É aconselhável iniciar a semeadura com a cultivar P98Y70 e terminar com a P99R01. Esta última, pelo maior ciclo, têm período juvenil maior que a primeira, logo adapta-se melhor a semeadura mais tardia. Inúmeros fatores ambientais, tais como: umidade, temperatura e fotoperíodo podem influenciar na produtividade de grãos (Guimarães et al., 2008). O alcance de altas produtividades da cultura depende da adaptação da mesma ao ambiente. Por sua vez, esta, depende de fatores como as condições climáticas e nutricionais satisfatórias e sem que haja incidência de pragas e doenças (Sediyama et al., 2009; Cruz et al., 2010).

\section{CONCLUSÕES}

As cultivares mais produtivas são a P98Y70 e a P99R01. A cultivar mais tardia é a P99R01. A cultivar MSOY 8352 RR não apresenta altura adequada para a colheita mecanizada. Todas as cultivares avaliadas apresentam altura de inserção de primeira vagem desfavorável à colheita mecanizada.

\section{AGRADECIMENTOS}

À FAPEMIG pela concessão de bolsas de Iniciação Científica.

\section{REFERÊNCIAS BIBLIOGRÁFICAS}

ALLEN, R. G.; PEREIRA, L. S.; RAES, D.; SMITH, M. Evapotraspiración del cultivo. Guías para la determinación de los requerimientos de agua de los cultivos Paper, 56. Roma: FAO - Utah State University, 2006. 323 p.

ALMEIDA, R. D. Divergência genética entre cultivares de soja $\mathrm{e}$ correlações entre suas características, sob condições de várzea irrigada, no sul do estado do Tocantins. 2008. $59 \mathrm{f}$. Dissertação (Mestrado em Produção Vegetal) - Universidade Federal do Tocantins, Gurupi. 


\section{AVALIAÇÃO AGRONÔMICA DE CULTIVARES DE SOJA SOB IRRIGAÇÃO NO SEMIÁRIDO MINEIRO}

BERNARDO, S.; SOARES, A. A.; MANTOVANI, E. C. Manual de Irrigação. 8 Ed. Viçosa: Editora UFV, 2006. $625 \mathrm{p}$.

BRACCINI, A. L.; MOTTA, I. S. SCAPIM, C. A.; BRACCINI, M. C. L.; ÁVILA, M. R.; MESCHEDE, D. K. Características agronômicas e rendimento de sementes de soja na semeadura realizada no período de safrinha. Bragantia, Campinas, v. 63, n. 1, p. 81-92, 2004.

CÂMARA, G. M. S.; SEDYAMA, T.; DOURADO NETO, D.; BERNARDES, M. S. Influence of photoperiod and air temperature on the growth, flowering and maturation of soybean (Glycine $\max ($ L.) Merrill). Scientia Agricola, v. 54, p. 149-54, 1997.

CARVALHO, E. R.; RESENDE, P. M.; OGOSHI, F. G. A.; BOTREL, E. P.; ALCANTRA, H. P.; SANTOS, J. P. Desempenho de cultivares de soja [Glycine max (L.) Merril] em cultivo de verão no sul de Minas Gerais. Ciência e Agrotecnologia, v. 34, n. 4, p. 892-899, 2010 .

CONAB - COMPANHIA NACIONAL DE ABASTECIMENTO. Acompanhamento da Safra Brasileira: Grãos Safra 2008/2009 Oitavo Levantamento. Disponível em: <http://www.conab.gov.br/conabweb/do wnload/safra/9graos_08.09.pdf. Acesso em: 10 de jun. 2010.

CRUZ, T. V.; PEIXOTO, C. P.; MARTINS, M. C.; PEIXOTO, M. F. S. P. Componentes de produção de soja em diferentes épocas de semeadura, no oeste da Bahia. Bioscience Journal, v. 26, n. 5, p. 709-716, 2010.

DHINGRA, O. D.; MENDONÇA, H. L.; MACEDO, D. M. Doenças e seu controle. In: SEDIYAMA, T. (Ed). Tecnologias de produção e usos da soja. Londrina: Mecenas, 2009. p. 133156.

ENDRES, V. C. Espaçamento, densidade e época de semeadura. In: EMPRESA BRASILEIRA DE PESQUISA AGROPECUÁRIA. Centro de Pesquisa Agropecuária do Oeste. Soja: recomendações técnicas para Mato Grosso do Sul e Mato Grosso (Circular Técnica, 3). Dourados: EMBRAPA, 1996. p. 82-85.

FIDELIS, R. R.; SILVA, J. C.; PELUZIO, J. M.; CAPPELLARI, D.; BARROS, H. B.; CARVALHO, G. L. Performance de cultivares de soja em diferentes épocas de semeadura na região do estado do Tocantins, safra 2006/2007. Amazônia: Ciência \& Desenvolvimento, v. 3 , n. 5, p. 165173, 2007.

GUIMARÃES, F. S.; REZENDE, P. M.; CASTRO, E. M.; CARVALHO, E. A.; ANDRADE, M. J. B.; CARVALHO, E. R. Cultivares de Soja [Glycine max (L.) Merrill] para cultivo de verão na região de Lavras - MG. Ciência e Agrotecnologia, v. 32, n. 4, p. 1099-1106, 2008.

JACOMINE, P. K. T.; CAVALCANTI, A. C.; FORMIGA, R. A.; SILVA, F. B. R.; BURGOS, N.; MEDEIROS, L. A.; LOPES, O. P.; MELO FILHO, H. R. L.; PESSOA, A. G. P.; LIMA, P. C. Levantamento exploratório: reconhecimento de solos do Norte de Minas Gerais - área de atuação da Sudene. Recife: EMBRAPASNLCS/SUDENE-DRN, 1979. p. 10-11.

NAVARRO JUNIOR, H. M.; COSTA, J. A. Expressão do potencial de rendimento de cultivares de soja. 
Pesquisa Agropecuária Brasileira, v. 37, n. 3, p. 275-279, 2002.

PICANÇO, M. C. Manejo Integrado das Pragas. In: SEDIYAMA, T. (Ed). Tecnologias de produção e usos da soja. Londrina: Mecenas, 2009. p. 119132.

RIBEIRO, A. C.; GUIMARÃES, P. T. G.; ALVAREZ V. V. H. Recomendação para o uso de corretivos e fertilizantes em Minas Gerais: 5 Ed. Viçosa: Comissão de Fertilidade do Solo do Estado de Minas Gerais, 1999.

RODRIGUES, O.; DIDONET, A. D.; LHAMBY, J. C. B.; BERTAGNOLLI, P. F.; LUZ, J. S. Resposta quantitativa do florescimento da soja à temperatura e ao fotoperíodo. Pesquisa Agropecuária Brasileira, v. 36, n. 3, p. 431-437, 2001.

SAEG - Sistema para Análises Estatísticas, Versão 9.1. Viçosa: Fundação Arthur Bernardes, 2007.

SEDIYAMA, T.; TEIXEIRA, C.; BARROS, H. B. Cultivares. In: SEDIYAMA, T. (Ed). Tecnologias de produção e usos da soja. Londrina: Mecenas, 2009. p. 77-92.

YOKOMIZO, G. K. Interação genótipos $x$ ambientes em topocruzamentos de soja tipo alimento com tipo grão. 1999. 170 f. Tese (Doutorado em Produção Vegetal) - Escola Superior de Agricultura Luis de Queiroz, Piracicaba. 